\title{
Application of Bayesian Network Methodology for Evaluating Industrial Control System
}

\author{
Jinsoo Shin ${ }^{1}$, Hanseong $\mathrm{Son}^{2}$, Soonghon $\mathrm{Kim}^{2}$ and Gyunyoung Heo ${ }^{1}$ \\ ${ }^{1}$ Kyung Hee University, 1732, Deoyeong-daero, Giheung-gu, Yongin-si, Gyeonggi-do, \\ 446-701, Republic of Korea \\ 2 Joongbu University, 201 Daehak-ro, Chubu-Myeon, Geumsan-gun, \\ Chungnam, 312-702, Republic of Korea \\ \{bigjoyman,gheo\}@khu.ac.kr, \{hsson,sgkim\}@joongbu.ac.kr
}

\begin{abstract}
Development in technology introduces novel kind of issues like cost optimization, system safety and cyber-attack. In order to solve these issues, sufficient information is needed but generally there is lack of information. We introduce the Bayesian network that is one of the most popular probabilistic methodologies to overcome the limitation of the issues. This paper presents two Bayesian network models related to system optimization and cyber security for specific industry control system to prepare the mobile environment.
\end{abstract}

Keywords: Bayesian network; System Optimization; Cyber Security

\section{Introduction}

The development of technology related to mobile systems such as personal digital assistant (PDA), mobile phone and laptop has influence on industries or workers. The characteristic of mobile device provides mobility and spacious working environ ment to users by using it [1-2]. There are a few common concerns between nuclear industry and mobile device such as standardization and cost optimization with advent of new technology, safety and cyber threats. Cost optimization is important from business point of view and has equal importance in both industries. In nuclear safety, one is interested in the safety of critical assets and there safe operation to keep public, worker and environment safe from hazardous radiation. Using the devices based on digital technology can introduce the some issues cyber security to industrial system, especially critical infrastructure like nuclear power plant. The system optimization issue is to find out the optimal configuration of the system, caused by modernization from analog to digital system or changing the system configuration, by comparison reliability of system with cost of system. The cyber security is very important issue on industrial facilities, especially critical infrastructure control system, because it influence to the hazard to the public safety as well as the finance loss. We need sufficient information to solve these issues, but generally there is lack of information. In order to overcome these problems, we introduce the Bayesian network methodology which is one of the most popular probabilistic methodologies based on Bayesian theory (in Chapter 2). Bayesian network $(\mathrm{BN})$ can overcome the problem about lack of information. Then, in this paper, we propose two models used the $\mathrm{BN}$ to represent that the $\mathrm{BN}$ methodology can be 
applied to these issues (in Chapter 3). One is the optimization model by using the BN to find out the optimal point between reliability and cost of system. And other is the cyber security evaluation model to find out vulnerability and optimal mitigation measure of cyber threat.

\section{Bayesian Network (BN)}

Bayesian network (BN) is a directed acyclic graph (DAG) of arc to represent the dependencies between nodes, which represent the variables, using the Bayesian theory. BN is composed of node, arc and node probability table (NPT) [3]. The arc represent the cause-and-effect relationship and NPT means the probability table that summarizes the occur probability between the causal relationship nodes. $\mathrm{BN}$ is useful to solve the problems like lack of information, posterior inference and changing from the qualitative to quantitative problem by learning new information on the conditional probability with calculation of the relationship between the posterior and prior probability.

\section{Modeling by Using BN}

$\mathrm{BN}$ can be one methodology for solving the lack of information issue or cyber security issue. In Chapter 3, we propose two represent model to find out optimal system configuration and vulnerability and optimal mitigation measure for cyber- attacks caused by mobile device, which applies to industrial control system (ICS). In this paper, the subject of application is restricted to nuclear reactor protection system (RPS) as ICS of critical infrastructure. RPS in nuclear industry is important to retain the safety state when an accident occurs to a nuclear facility. Then, two models are developed by using $\mathrm{BN}$. These models are significant with the introduction of digital, like mobile, I\&C system.

\subsection{Optimal Model for RPS Architecture}

To find out optimal configuration of system, we make several configuration of nuclear reactor protection system (RPS) as one of ICS and an optimization model to determine optimal instrumentation and control (I\&C) architecture configuration in terms of availability and cost. The four cases with different architecture formation for RPS were developed with single and double redundancy of bi-stable modules, coincidence processor module, and safety or protection circuit actuation logic. The architecture configurations are transformed to reliability block diagram based on logical operation and function of modules. The $\mathrm{BN}$ models for four configurations were developed to get the availability analysis, one configuration is shown in Fig. 1, by using AND gate, OR gate and N-out-of-M gate as BN functions [4]. Cost estimation model was proposed for the architecture configurations, respectively. The BN model can be used to calculate the system reliability. For cost estimation, a numeric multiplier of $\mathrm{X}$ arbitrary units was assigned to each component based on experts and designers judgment and cost for each configuration was calculated. Reliability index, which means the increase of reliability per unit of cost, is proposed by calculation of cost and availability with each configuration and a configuration of architecture that gives highest availability with maintaining low cost of manufacturing can be identified. It was found that reliability can be enhanced at the rate of 5E-05 per X unit of cost for this study. 


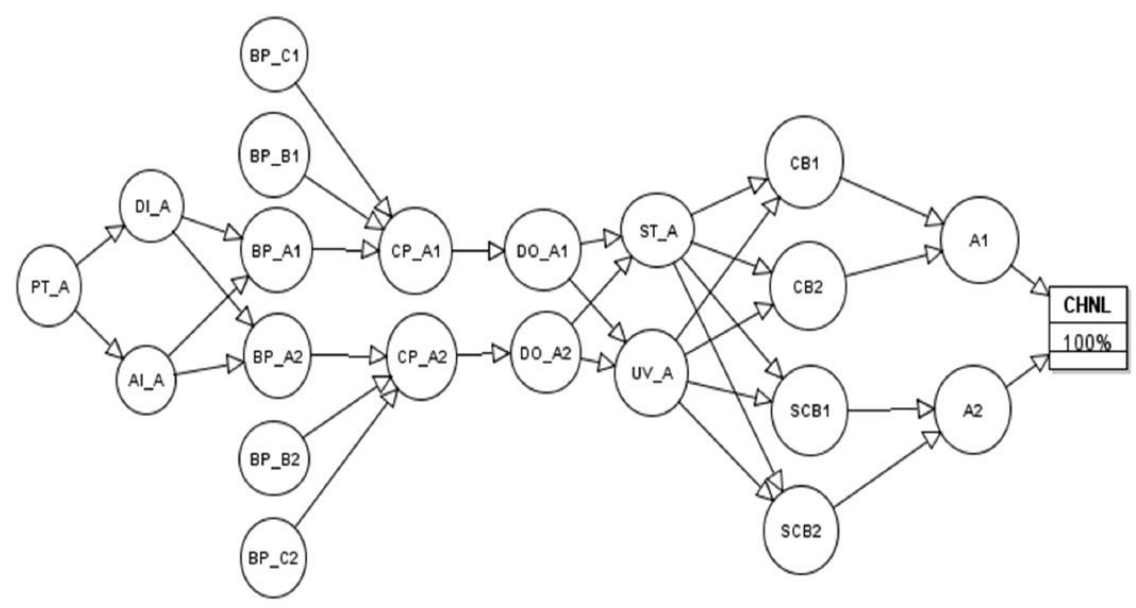

Figure 1. BN model of I\&C architecture with redundancy in BP \& CP

\subsection{Cyber Security Risk Model for RPS Architecture}

Mobile like PDA, mobile phone and laptop technology give usability to user during maintain period of component, device and system. The cyber-attack is emphasized as new critical threat caused by applying digital device into ICS. Cyber security against cyber-attack is difficult to analysis and modelling due to lack of information. We suggest the cyber security risk model for RPS with the BN to overcome these problems by calculating of the relationship between the posterior and prior probability [5-6]. The model utilizing the benefit of BN can analyze the risk that cyber-attack occurs at RPS. It can be utilized for the quantitative analysis by the proposed measure, cyber security risk as well as for various qualitative analyses. Cyber security risk model is composed of the activity-quality analysis model and the architecture analysis model. The activityquality analysis model was proposed to check how people and/or organization comply with the cyber security regulatory guide. It helps to analyze the relationships of the activity-quality checklists and their influences to cyber security. The architecture analysis model was also developed for the RPS as an illustrative purpose. For the definition of the critical cyber-attack scenarios on RPS of nuclear industry, the vulnerabilities and mitigation measures were analyzed. Then, the two models, activityquality and architecture analysis models, were integrated to cyber security risk model by using BN. Then, several analyses were performed by using the cyber security risk model for confirming the model. The analysis of the vulnerability and optimal mitigation measure against the vulnerability was performed with the assumption that a cyber-attack occurs to a maintenance and test processor in the RPS. In this analysis, the vulnerabilities and the mitigation measures were analyzed with the assumed cyberattack to RPS. If a cyber-attack occurs at a system scale, it is important to have confidence on which component is the key element corresponding to the attack situation. This analysis proved that the developed model could provide this kind of information through the back propagation feature of the BN. Finally, the analysis of the cyber security risk and the optimal mitigation measures regarding vulnerabilities was performed on RPS. This analysis infers that the use of the cyber security risk model makes it possible to create simulated penetration test scenarios for future directions. 


\section{Conclusions}

For preparing the introduction of the latest devices based on digital interface like mobile on critical infrastructure facilities, we suggest the BN methodology and the two models based on the methodology to demonstrate the usefulness of the $\mathrm{BN}$ for specific ICSs. One is the model for the optimization of system configuration in view of reliability and cost. This model was developed for RPS and demonstrated the usefulness of the BN. Using this model, we could find out an optimal configuration by analyzing the reliability and cost of each changing configuration. The other is the cyber security evaluation model, which is also based on the $\mathrm{BN}$, to analyze the vulnerabilities and the optimal mitigation measures for RPS architecture. The model found out the critical vulnerabilities and the optimal mitigation measures against the vulnerabilities for several assumed scenarios by using the $\mathrm{BN}$ function of back propagation calculation. The studies using these two models infer that the BN methodology can be applied to other systems like mobile systems for the same purposes.

\section{Acknowledgements}

This work was supported by Advanced Research Center for Nuclear Excellence (ARCNEX) program funded by the Ministry of Education, Science and Technology (Grant Number: 2012-055432)

\section{References}

[1] H. C. Lee and G. B. Lee, "Prototype of Operator Interface for Monitoring and Controlling in the Mobile Control Room", Proceedings of the 2013 ACM international conference on interactive tabletops and surfaces, (2013), pp 305-308.

[2] K. Ji and T. J. Li, "Detection and Analysis of Unidirectional Licks in Mobile Ad Hoc Network Under Nuclear Power Plants Environment", Emerging Technologies for Information Systems, Computing, and Management Lecture Notes in Electrical Engineering, vol. 236, (2013), pp. 971-878.

[3] D. Heckerman, "A tutorial on learning with Bayesian networks, (1996).

[4] K. Rahman, J. S. Shin, G. Y. Heo and H. S. Son, "Reliability Analysis of I\&C Architecture of Research Reactors Using Bayesian Networks”, Korean Nuclear Society Autumn Meeting, Gyeongju, Korea, (2013) October 23-25.

[5] J. S. Shin, H. S. Son, K. Rahman and G. Y. Heo, "Development of Cyber Security Evaluation Model Using Bayesian Networks", Reliability Engineering and System Safety (submission, 2013).

[6] J. S. Shin, H. S. Son and G. Y. Heo, "Cyber Security Risk Analysis Model Composed with Activity-quality and Architecture Model, International Conference on Computer, Networks and Communication Engineering, Beijing, China (2013) May 23-24, pp. 609-612.

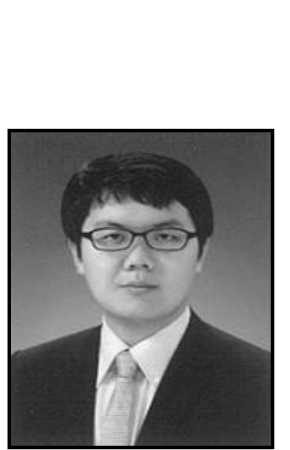

\section{Authors}

\section{Jinsoo Shin}

Jinsoo Shin received the B.S., and M.S. degrees in department of nuclear engineering, Kyung Hee University, Suwon, Korea, in 2012, and 2013, respectively. Currently, he is on the Ph.D. program at Mainformatics Laboratory in Kyung Hee University. His research interest is the application of Bayesian network methodology for nuclear instrumentation and control system design, cyber security evaluation, and anomaly diagnosis. 


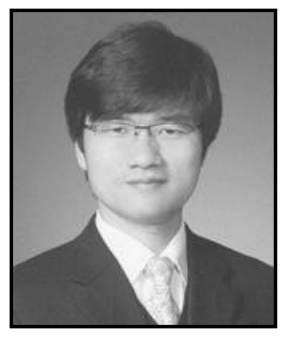

\section{Hanseong Son}

Hanseong Son received a Ph.D. degree from Korea Advanced Institute of Science and Technology in Nuclear Engineering in 2000. He has been working as an assistant professor in Joongbu University from March 2008. His research interests include software engineering, software reliability, cyber security, and so forth.

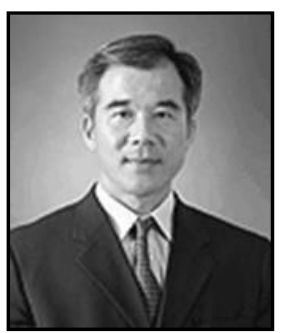

\section{Soonghon Kim}

Soonghon Kim received a Ph.D. degree from Chonbuk National University, Seoul Korea, in Computer Engineering in 1999. He has been working as a Professor in Joongbu University from March 1995. His research interests include Ubiquitous Computing, Distributed Computing, Database Integrity, Cryptographic Protocol, A methodology of Software Development, Software Evaluation, Networks and so on.

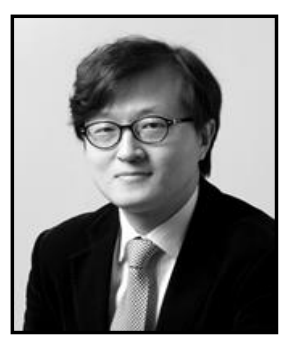

\section{Gyunyoung Heo}

Gyunyoung Heo received the B.S., M.S., and Ph.D. degrees in nuclear and quantum engineering, Korea Advanced Institute of Science and Technology, Daejeon, Korea, in 1990, 1999, and 2004, respectively. Currently, he is a Associate Professor and the director of Mainformatics Laboratory in Kyung Hee University, South Korea. His research interest is the analysis of performance and reliability for industrial plants on the basis of intelligent data processing, including statistics and artificial intelligence. His major activities are the development of plant simulation codes and operator aid systems, probabilistic risk assessments for nuclear facilities, and the invention of state analysis techniques combining statistical methods and heuristic methods. 
International Journal of Control and Automation Vol.7, No.3 (2014) 